\title{
Le réchauffement climatique à l'ère de la covid-19 dans la Province du Haut-Katanga
}

\author{
Par MWENZE MINZA Martin* et KALAMA KASEBA Odon**
}

\section{INTRODUCTION}

«Les changements climatiques sont l'un des défis déterminants du XXI ${ }^{\mathrm{e}}$ siècle. Il s'agit d'un problème mondial et s'y attaquer nécessitera une action à l'échelle mondiale. Les gouvernements du monde entier se sont engagés à collaborer afin de limiter le réchauffement de la planète, reconnaissant que les risques liés au climat augmentent avec l'ampleur du réchauffement et les changements associés au climat. L'Entente de Paris en vertu de la Convention-cadre des Nations Unies sur les changements climatiques, qui est entrée en vigueur le 4 novembre 2016, a établi un objectif de maintenir l'augmentation de la température mondiale entre $1,5{ }^{\circ} \mathrm{C}$ et $2{ }^{\circ} \mathrm{C}$ au-dessus des niveaux préindustriels, de même qu'un engagement à participer dans la planification de l'adaptation ainsi que sa mise en œuvre. Une action collective en vue de l'objectif de la température mondiale est mise en œuvre.

Cependant, il est reconnu que cet objectif servira seulement à réduire, et non à éliminer les risques et les impacts des changements climatiques. Les gouvernements et les citoyens doivent comprendre la façon dont les changements climatiques peuvent les toucher, afin de planifier et de se préparer pour les défis que les changements climatiques apporteront ${ }^{1}$.

Comprendre les changements climatiques et leurs conséquences s'inspire des sciences physiques, biologiques et sociales. Les recherches en cours dans ces domaines mènent vers un ensemble constamment grandissant de documents scientifiques publiés concernant les changements climatiques. Evaluer cette base de connaissances grandissante et communiquer la façon dont la compréhension des changements climatiques a évolué est difficile, surtout parce qu'il y a un vaste public pour cette information. Les impacts du changement climatique sont une préoccupation pour les personnes, les collectivités, les entreprises et les gouvernements, que ce soit à l'échelle locale et régionale ou à l'échelle nationale et internationale.

La République Démocratique du Congo (RDC) figure, depuis juin 1992, parmi les plus de 185 pays qui font parties de la Convention Cadre des Nations Unies sur les Changements Climatiques (CCNUCC). C'est à ce titre qu'elle a entrepris depuis lors, une série d'activités

* Chef de Travaux à la Faculté de Sciences politiques et administratives de l'Université de Lubumbashi.

** Assistant à la Faculté de sciences politiques et administratives de l'Université de Lubumbashi.

1 Bush, E. et G. Flato, rapport sur l'environnement et le changement climatique, Ottawa, Ontario, 2019, p. 7-23. 
scientifiques conformes aux engagements auxquels elle a souscrit, à savoir l'Inventaire de ses émissions des Gaz à Effet de Serre (GES) pour l'année 1994, lequel a abouti à la Communication Initiale Nationale sur les changements climatiques présentée en 2002 à New Delhi, lors de la $8^{\text {eme }}$ Conférence des Parties (CoP8).

Dans le même ordre d'idée, le pays a entrepris d'élaborer son premier Programme d'Action National d'Adaptation aux changements climatiques (PANA). Le climat d'un pays constitue une de ses ressources naturelles fondamentales. En effet, tous les facteurs climatiques sont des facteurs écologiques. Or, ce sont ces derniers qui déterminent un écosystème donné.

Cependant, avec l'entrée de la terre dans l'ère dite « de l'Anthropocène », les activités humaines ou la Technosphère provoquent des changements climatiques. La province du Haut-Katanga étant l'une des vingt-six provinces de la République Démocratique du Congo, se trouve embarquée dans ce navire.

Cette étude a été réalisée dans une approche participative qui s'est articulée sur des échanges et briefings instructifs et d'orientation sur les questionnements et la compréhension de la covid-19.

Aussi, sommes-nous persuadé que la présente étude contribue, tant soit peu, à renforcer les mécanismes de lutte contre le réchauffement climatique.

Beaucoup de maladies seraient liées aux facteurs climatiques. A titre d'exemples, le paludisme, la rougeole, la coqueluche, la dingue, les accidents vasculaires cérébraux (AVC), la fièvre typhoïde, le coronavirus. Cependant, l'impact notable de ces facteurs sur la santé est, à ce jour, difficilement quantifiable.

La variabilité climatique la plus souvent prise en compte dans les relations changement climatique -santé dans la région intertropicale c'est l'influence des événements ENSO (E1 Niño Southern Oscillation) sur l'émergence des maladies.

Ainsi, l'approche adoptée dans le cadre de l'étude de la vulnérabilité et de l'impact des changements climatiques sur la santé en République Démocratique du Congo en général et dans la province du Haut-Katanga en particulier est de nature "épidémiologique", par la recherche des corrélations statistiques qui s'établissent entre quelques maladies de cette nature et certains paramètres climatiques, notamment la température et les précipitations, l'indice climatique SOI pour l'ENSO.

\section{A. NOTION SUR LES CHANGEMENTS CLIMATIQUES}

Les changements climatiques sont causés par les modifications de l'atmosphère qui résultent de sa transformation chimique par les gaz à effet de serre (GES). Cette perturbation de l'équilibre atmosphérique s'exprime par une augmentation des températures moyennes sur Terre, modifiant ses caractéristiques physiques, chimiques et biologiques. ${ }^{2}$

2 https://www.naturequebec.orglressourceslfichierslAgriculturelfermeszerocarbone 20IIITXTII-03 ref ChangClimat.pdf consulté le 10 décembre 2020. 
Les impacts sur l'environnement sont multiples, importants et de plus en plus fréquents : sécheresses, fonte des glaciers et de la glace de mer, élévation du niveau des océans, tempêtes tropicales. Ils affectent l'ensemble de la population mondiale et la biodiversité planétaire. Les activités humaines sont les principales responsables des changements climatiques actuels et de leurs impacts sur l'environnement. En effet, selon le Groupe d'experts intergouvernemental sur l'évolution du climat (GIEC), le réchauffement climatique est bien réel et l'activité humaine en est responsable, par l'émission de GES.

Par ailleurs, « au cours du siècle dernier, un réchauffement moyen de la température à la surface de la terre de $0,74{ }^{\circ} \mathrm{C}$ a été observé, alors qu'il était prévu que la température moyenne n'augmenterait que de $0,6^{\circ} \mathrm{C}$ (GIEC, 2008). Les prévisions pour 2100 sont encore plus alarmantes, avec une augmentation prévue de la température moyenne de 2 à $4,5{ }^{\circ} \mathrm{C}$ $\left(\right.$ GIEC, 2008) ». ${ }^{3}$

\section{LES GAZ A EFFET DE SERRE}

Les GES sont naturellement présents dans l'atmosphère. Ces gaz forment une couche autour de la Terre, lui permettant de conserver sa chaleur : c'est l'effet de serre. En effet, le soleil réchauffe la Terre qui, par la suite, réémet une partie de sa chaleur vers l'espace. Les GES présents dans l'atmosphère emprisonnent une partie de cette chaleur, l'empêchant de retourner dans l'espace. Ce phénomène permet de conserver des températures moyennes de $15{ }^{\circ} \mathrm{C}$ sur notre planète. Sans cela, la température y serait d'environ $-18{ }^{\circ} \mathrm{C}$, ce qui ne permettrait pas la présence de la vie telle que nous la connaissons (Environnement Canada, 2009).

Les GES ne contribuent pas tous à l'effet de serre de façon équivalente. En effet, leur pouvoir de réchauffement global (PRG) et leur durée de vie dans l'atmosphère sont différents. Le PRG correspond à la capacité du gaz à conserver la chaleur autour de la terre, en la renvoyant vers le sol. Le PRG des GES s'évalue en les comparant au PRG du CO2, le gaz de référence. Le CO2-équivalent (CO2e) est donc une mesure des GES, qui permet une comparaison de l'impact de chacun des GES, selon le PRG. Par exemple, pour une même quantité, le $\mathrm{N} 2 \mathrm{O}$ réchauffe 310 fois plus l'atmosphère que le $\mathrm{CO} 2$, donc $1 \mathrm{~kg}$ de $\mathrm{N} 2 \mathrm{O}$ émis correspond à $310 \mathrm{~kg}$ de $\mathrm{CO} 2 \mathrm{e}$.

Le temps de séjour dans l'atmosphère représente le temps de résidence moyen des GES. On constate que les émissions de GES d'aujourd'hui contribueront aux changements climatiques pendant des dizaines, voire des centaines d'années.

L'augmentation accélérée des concentrations de GES dans l'atmosphère amplifie le phénomène naturel d'effet de serre. La concentration des GES dans l'atmosphère a été pratiquement constante pendant des milliers d'années, et a permis le développement de la vie telle qu'on la connaît. Cependant, cet équilibre est précaire et les émissions actuelles de GES

3 https://www.naturequebec.orglressourceslfichierslAgriculturelfermeszerocarbone 20IIITXTII-03 ref ChangClimat.pdf, consulté le 10 décembre 2020. 
sont supérieures à tout ce que la planète a connu depuis 650000 ans (GIEC, 2008). Le développement des activités humaines modernes, dont le transport, l'industrie, la déforestation et l'agriculture, est responsable de l'émission massive de trois principaux GES : le dioxyde de carbone $\left(\mathrm{CO}_{2}\right)$, le méthane $\left(\mathrm{CH}_{4}\right)$ et le protoxyde d'azote $\left(\mathrm{N}_{2} \mathrm{O}\right)$. La concentration de ces trois gaz a considérablement augmenté depuis l'ère industrielle : de $35 \%$ pour le $\mathrm{CO}_{2}$, de $148 \%$ pour le $\mathrm{CH}_{4}$ et de $18 \%$ pour le $\mathrm{N}_{2} \mathrm{O}$ (MDDEP, 2010). Ce déséquilibre modifie les températures à la surface de la Terre, causant de nombreux impacts à l'environnement.

Un avertissement a été émis par les scientifiques du climat : l'augmentation des températures moyennes doit être limitée à $2{ }^{\circ} \mathrm{C}$, sans quoi le climat risque de s'emballer. Malgré cet avertissement, et bien que les changements climatiques soient déjà perceptibles, les émissions de GES des pays développés augmentent continuellement, année après année. Au Québec, les émissions ont augmenté entre 1990 et 2007, mais en 2008, elles étaient sous la barre de 1990. Toutefois, ces réductions sont surtout attribuables à un ralentissement du secteur industriel entre 2007 et 2008.

Tout un travail reste à faire pour réduire nos émissions de GES et ainsi diminuer nos risques d'expositions aux impacts des changements climatiques, même si nous savons aussi que nous devrons également nous y adapter.

\section{EFFETS DES CHANGEMENTS CLIMATIQUES}

L'augmentation des GES dans l'atmosphère a déjà provoqué des modifications du climat mesurables. On en observe déjà les impacts, avec des événements qui se produisent à une vitesse et une ampleur plus élevées que prévues. Les changements climatiques affectent de différentes façons l'environnement, la planète et les humains qui l'habitent.

Voici un aperçu de ce qui est déjà observé, ainsi que les prévisions relatives aux changements climatiques :

\section{L'eau}

Les changements climatiques sont responsables de la fonte des glaces, dont le rythme s'est accéléré depuis les 15 dernières années. La fonte massive des glaces augmente la masse d'eau, occasionnant une élévation du niveau de la mer. Les élévations prévues sont de 9 à $88 \mathrm{~cm}$ entre 1990 et 2100 . Cette situation aura des conséquences néfastes sur les basses terres côtières où vit une très grande partie de la population mondiale. Déjà, en 2008, les désastres reliés au climat auraient causé le déplacement de 20 millions de personnes ${ }^{4}$. De plus, les ressources en eau potable seront affectées. Par exemple, l'augmentation des températures pourraient occasionner une baisse considérable du niveau d'eau des Grands Lacs, en raison d'une plus grande évaporation. Les Grands Lacs, en plus d'être l'un des principaux tributaires du fleuve Saint-Laurent, représentent la plus importante source d'eau

4 IOCHA and IDMC, 2009. 
douce au monde. Cette diminution de la quantité d'eau potable pourrait être problématique et s'avérer une cause majeure de conflits entre les nations ${ }^{5}$. La qualité de l'eau des lacs sera aussi à surveiller, car la prolifération des algues bleu-vert pourrait s'intensifier dans le contexte des changements climatiques.

\section{La précipitation, la sécheresse et le climat extrême}

Les changements climatiques bouleversent déjà les patrons de précipitations. Ces bouleversements seront accompagnés d'une augmentation de la fréquence et de l'intensité des événements climatiques extrêmes : sécheresses, inondations, canicules, pluies fortes et abondantes, tornades... Ces événements climatiques, souvent à l'origine de catastrophes, pourraient se produire plus fréquemment dans le futur ${ }^{6}$. Au Québec, les prévisions indiquent une augmentation des précipitations pouvant aller jusqu'à $25 \%$ au printemps et $32 \%$ en hiver, d'ici $2050^{7}$. Malgré ces prévisions, il n'existe aucune certitude concernant le taux d'enneigement de nos hivers, car les précipitations pourraient aussi se produire sous forme de pluies.

\section{La santé}

La plupart des scientifiques reconnaissent maintenant que les changements climatiques auront de graves conséquences sur la santé humaine. Au Canada, plusieurs impacts sur la santé ont été identifiés, dont l'augmentation des maladies respiratoires comme l'asthme, des cancers de la peau, des malaises et des mortalités liées à des chaleurs intenses ${ }^{8}$. De plus, les changements climatiques peuvent amplifier le phénomène d'îlots de chaleur en milieu urbain ${ }^{9}$, un phénomène qui a des impacts sur la santé, allant des crampes de chaleur à la syncope de chaleur, et de l'épuisement par la chaleur au coup de chaleur ${ }^{10}$. Aussi, une augmentation des maladies transmises par les insectes et les animaux transmises est à prévoir.

Notons la nuance entre le climat et la météo :

La météo et le climat sont deux façons différentes d'observer les températures sur la terre. Le réchauffement climatique annoncé est une tendance à long terme de l'augmentation des températures moyennes. Ces prévisions ne doivent pas être confondues avec les prévisions météorologiques quotidiennes, saisonnières ou annuelles. En effet, la météo est une observation à court terme, et a toujours été variable. Par exemple, bien que les prévisions annoncent un réchauffement climatique et une augmentation des précipitations

5 Environnement Canada, 2002.

6 Environnement Canada, 2002.

7 Bourque et Simonet, 2007.

8 Santé Canada, 2008.

9 Giguère, 2009.

10 Denis, 2010. 
en hiver au Québec, le prochain hiver pourrait très bien être particulièrement froid et sec. Ce sont les observations à long terme qui permettent de constater une variation du climat.

\section{Le secteur agricole}

Concernant l'agriculture, les changements climatiques pourraient avoir des impacts autant négatifs que positifs. En général, une augmentation des températures moyennes et un allongement de la saison de croissance devraient occasionner un accroissement potentiel du rendement des cultures. De même, ces modifications devraient rendre possible la production de cultures adaptées à des températures plus élevées ${ }^{11}$.

À l'inverse, la protection hivernale qu'offre la couverture neigeuse aux cultures pérennes pourrait être affectée par des hivers plus doux et moins enneigés. De plus, des automnes plus doux pourraient diminuer les conditions optimales à l'endurcissement et causer plus de dommages aux plantes fourragères ${ }^{12}$.

Les risques d'invasion par les insectes ravageurs pourraient augmenter et la répartition des espèces pourrait être modifiée au cours des prochaines années, en raison de conditions climatiques plus propices ${ }^{13}$. Par exemple, des observations confirment que la chrysomèle des racines de l'ouest et la tordeuse orientale du pêcher, qui étaient présentes au sud du Québec, progressent maintenant vers le nord. Dorénavant, la présence de certains insectes est observée sur une plus longue période, ce qui, par conséquent, implique que le dépistage devra débuter plus tôt en saison.

Les mauvaises herbes bénéficieront également de nouvelles conditions favorables provoquées par les changements climatiques, favorisant l'expansion de leur aire de développement. De plus, selon certaines études, les mauvaises herbes auraient de meilleures capacités d'adaptation aux modifications du climat que les cultures.

L'adaptation de l'agriculture aux nouvelles conditions liées aux changements climatiques sera nécessaire. Bien sûr, beaucoup de défis attendent les producteurs, mais ceux-ci ont la possibilité de s'adapter aux changements climatiques en introduisant de nouvelles variétés de cultures ou de nouveaux types de productions, en assurant une meilleure protection des sols et de meilleurs conditions hydriques. Plus vite les producteurs agricoles sauront s'adapter aux changements climatiques, plus ils en réduiront les impacts négatifs sur leur exploitation. En effet, les actions de lutte aux changements climatiques réalisées maintenant sont plus avantageuses que les adaptations nécessaires qui seront imposées par les impacts.

11 Bélanger, 2002.

12 Ibidem.

13 Denis, 2010.

Roy, 2002. 


\section{LES ENJEUX RELIÉS AUX CHANGEMENTS CLIMATIQUES ${ }^{14}$}

La lutte aux changements climatiques est devenue une priorité de la communauté internationale lors de la signature du Protocole de Kyoto en 1997. À cette occasion, plusieurs pays se sont engagés à réduire, d'ici 2012, leurs émissions de GES de 5,2\% par rapport au niveau de 1990, année de référence. Le Québec s'est fixé comme objectif de réduire de $6 \%$ ses émissions de GES par rapport aux émissions de 1990, d'ici 2012. Il a récemment annoncé de nouveaux objectifs, de diminution de ses émissions de $20 \%$ sous le seuil de 1990 , pour 2020 .

\section{REGARD SUR LE CLIMAT EN REPUBLIQUE DEMOCRATIQUE DU CONGO}

Le climat de la RDC est diversifié. Il est de type équatorial, chaud, humide au centre du pays, et tropical vers le sud et le nord. La pluviométrie est régulière et abondante en RDC $(1.545 \mathrm{~mm} / \mathrm{an}$ en moyenne), mais varie dans le temps et dans l'espace (de 800 à $1800 \mathrm{~mm}$ ). En général, on distingue quatre types de climat à travers le pays ${ }^{15}$ :

- Climat équatorial : Il est rencontré au niveau de la cuvette centrale où les températures mensuelles de mois de mai et juillet peuvent respectivement atteindre $27^{\circ}$ et $25^{\circ} \mathrm{C}$. L'amplitude thermique y est inférieure à $1^{\circ} \mathrm{C}$. Les précipitations annuelles varient entre 1.600 et $2.000 \mathrm{~mm}$. Sous ce climat, la saison sèche dure moins de 2 mois;

- Climat tropical humide : Les températures de mois de mars et juillet peuvent respectivement atteindre $28^{\circ}$ et $21^{\circ} \mathrm{C}$. L'amplitude thermique varie entre 1 et $3^{\circ} \mathrm{C}$. Les précipitations annuelles, sont généralement comprises entre 1.200 et $1.600 \mathrm{~mm}$. La durée de la saison sèche varie entre 2 et 4 mois;

- Climat tropical à tendance aride : Les températures du mois de mars et juillet peuvent se situer entre 28 et $22^{\circ} \mathrm{C}$ à l'Ouest (Bas-Congo) et celle de novembre et juillet entre 24 et $16^{\circ} \mathrm{C}$ au Sud-est (Sud Katanga). L'amplitude thermique annuelle est supérieure à $3^{\circ} \mathrm{C}$ et peut même dépasser $5^{\circ} \mathrm{C}$. Les précipitations annuelles sont généralement comprises entre 1.000 et $1.200 \mathrm{~mm}$. La durée de la saison sèche est supérieure à 4 mois et peut atteindre 6 mois ou plus;

- Climat de montagne : On le rencontre généralement à l'Est du pays et il est tempéré par l'altitude. Les températures d'octobre et de juillet varient entre 25 et $20^{\circ} \mathrm{C}$. L'amplitude thermique est proche de $4^{\circ} \mathrm{C}$, les précipitations annuelles dépassent $1.000 \mathrm{~mm}$ dans le Nord Katanga et $1.200 \mathrm{~mm}$ à l'Est et au Nord-est du pays.

$14 \mathrm{https} / / /$ www.naturequebec.orglressourceslfichierslAgriculturelfermeszerocarbone 20IIITXTII-03 r efChangClimat.pdf, consulté le 10 décembre 2020.

15 République Démocratique du Congo, Ministère de l'Environnement, Conservation de la Nature et Tourisme, seconde communication nationale à la convention cadre sur les changements climatiques, données de l'inventaire des émissions et de séquestration des gaz à effet de serre (GES) de chaque secteur socio-économique entre 1999 et 2004 ainsi qu'une étude de la vulnérabilité du pays par rapport aux changements climatiques et des mesures d'adaptation des secteurs forêts, agriculture, zone côtière, eau et santé, p.59 (212) 


\section{B. ETAT DES LIEUX EN MATIERE DES CHANGEMENTS CLIMATIQUES DANS LE HAUT-KATANGA}

\section{Causes et conséquences}

Voici les causes à base des changements climatiques :

- Augmentation des gaz à effet de serre (GES);

- Mauvaise gestion des essences forestières (déboisement abusif et déforestation);

- Grande concentration de la population/ mauvaise urbanisation;

- Mauvaises affectations et utilisation des terres et des sols;

- Utilisations des terres et des sols;

- Procédés industriels non appropriés

- Faibles institutions de suivi;

- Faibles lois et cadres habilitants;

- Mauvaises pratiques de gestion des terres et des déchets;

- Faiblesses de l'éducation du public et de la sensibilisation...

Voici les effets à base des changements climatiques :

- Renforcement des inversions de température, plus particulièrement pendant la saison sèche;

- Augmentation du forcing thermique, d'où la canicule;

- Perturbation du régime pluviométrique (raccourcissement de la saison des pluies et allongement de la saison sèche, rareté des pluies, réduction du nombre des jours de pluie, augmentation des pluies intenses (d'où les inondations), diminution des eaux dans les aquifères, dégradation des terres, érosions ravinantes et envasement du réseau hydrographique);

- Perturbation du cycle hydrologique (étiage de plus en plus prononcé et assèchement des rivières);

- Perturbation des écotopes (consumation des forêts (feu) et intensification des phénomènes météorologiques extrêmes (ouragan, tornade, typhon).

\section{Inventaire des capacités thématiques à renforcer}

1. Au plan Institutionnel

- Renforcer le rôle du Comité National des Changements climatiques en définissant clairement sa mission, en le structurant judicieusement et en 'associant aux prises de décisions impliquant le climat;

- Créer des Secrétariats provinciaux des changements climatiques tout en leur assignant des missions spécifiques claires et précises;

- Introduire des cours de météorologie, climatologie et hydrologie générales dans les filières d'enseignement supérieur et universitaire des sciences naturelles; 
- Veiller à la qualité des procédés industriels en place et/ou à implanter au pays;

- Introduire des émissions audio-visuelles des thèmes climatiques dans des grilles des programmes;

- Participer régulièrement et efficacement à toutes les rencontres internationales sur le climat;

- Veiller à la production régulière des communications nationales au titre de la CCCC.

2. Au plan provincial : Action de contrôle, surveillance et lutte contre les pollutions Cette action va couvrir les activités suivantes :

- Monitoring sur la qualité de l'eau;

- Création des structures de prévention et de lutte contre les pollutions;

- Contrôle et surveillance des rejets et effluents industriels et des déchets municipaux;

- Contrôle et surveillance des pollutions par les navires;

- Mise en œuvre du plan provincial d'urgence de lutte contre les déversements des hydrocarbures.

3. Au plan Communautaire

- Evaluer régulièrement la vulnérabilité aux changements climatiques par provinces;

- Inventorier les connaissances endogènes sur le climat;

- Promouvoir l'approche participative par la sensibilisation et l'éducation de la population. Sensibiliser les communautés de base au sujet des changements climatiques surtout sur ceux les concernant plus directement;

- Organiser des sessions de formations thématiques en faveur des groupes cibles.

4. Au plan individuel

- Améliorer la capacité des individus à gérer et à protéger l'environnement;

- Améliorer la capacité des individus à travailler en tant qu'individus au sein des organisations et de la société;

- Changer les mentalités, la connaissance, le comportement et les actions des individus;

- Sensibiliser régulièrement les individus sur des questions liées aux changements climatiques de leurs milieux.

\section{EVALUATIONS INTERSECTORIELLES DES CAPACITES ET DE LA LOGISTIQUE A RENFORCER}

1. Au plan Intersectoriel

- Créer des « environnements propices » pour une meilleure gestion de l'environnement dans tous les secteurs de la société; 
- Mener des recherches multidisciplinaires en vue de répondre efficacement à ces problèmes;

- Améliorer le cadre général politique en vue de favoriser des échanges d'informations sectorielles;

- Promouvoir la participation de tous les secteurs de la société à la réalisation des objectifs environnementaux;

- Identifier les problèmes environnementaux clés;

- Améliorer la capacité d'acquisition et d'échange des connaissances (données) par secteur.

2. Au plan logistique

- Réhabiliter le réseau des collectes de données météorologiques, climatologiques et hydrologiques à travers la province;

- Equiper le Secrétariat provincial des changements climatiques en matériel informatique pour un traitement et un stockage adéquats des données;

- Doter le Secrétariat provincial des changements climatiques d'un minimum financier, en mobilier et en immobilier pour lui faciliter son travail;

- Créer une unité spéciale de contrôle des technologies en place et à importer.

\section{RECHERCHE ET OBSERVATION SYSTEMATIQUE SUR LES CHANGEMENTS CLIMATIQUES}

\section{Cadre institutionnel de recherche et d'observation systématique du climat}

Les institutions d'observation des paramètres climatiques relèvent de plusieurs ministères. Leurs compétences dans cette mission d'observation sont plus ou moins étendues selon le cas. Les institutions qui effectuent les observations des paramètres climatologiques ou hydrologiques pour le besoin de l'accomplissement de leurs activités statutaires sont :

L'Agence nationale de Météorologie et de Télédétection par Satellite (METTELSAT), service météorologique et hydrologique national, aux termes de l'OMM est le seul à avoir pour mission l'observation météorologique dans toutes ses composantes. Elle a aussi la charge de l'hydrologie opérationnelle ainsi que de l'observation des ressources naturelles par satellite. Institution publique par excellence en matière du suivi des changements climatiques, elle a grandement manifesté son intérêt pour l'ANCR. De besoins énormes en matière de formation du personnel, d'acquisition des nouvelles technologies et de diffusion des données météorologiques et climatologiques sont en réalité un casse-tête pour l'institution.

- L'Institut National pour les études et la Recherche Agronomiques (INERA) possède un réseau d'observation agro météorologique.

- La Régie des Voies Aériennes (RVA) est un prestataire de météorologie aéronautique. 
- La Régie des Voies Fluviales (RVF) gère un réseau hydrologique pour la mesure du débit et de la hauteur d'eau des cours d'eau qu'elle exploite pour la navigation.

- La Congolaise des Voies Maritimes (CVM) assume la même responsabilité dans le bief maritime du fleuve Congo.

- Le Service National de l'Hydraulique Rural (SNHR) : est une direction au sein du Ministère de l'Agriculture et du Développement rural qui a pour rôle de fournir de l'eau potable aux habitants des compagnes en quantité et en qualité suffisante et à une distance qui réduise la corvée, d'où le besoin d'évaluation des ressources d'eau souterraine et d'observation visant essentiellement à déterminer la présence de l'eau en vue de son exploitation.

- La Société Nationale de l'Electricité (SNEL) et la REGIDESO, effectuent également, en collaboration avec le ministère de l'énergie et des ressources hydrauliques, des mesures sur le potentiel hydroélectrique et de captage d'eau des sites sur les rivières congolaises.

A part l'INERA, le SNHR et la SNEL qui sont placés respectivement sous la tutelle des ministères de la Recherche scientifique, de l'Agriculture et de l'Energie, les autres institutions opèrent sous la tutelle du Ministère des Transports et des Voies de Communication.

\section{Système Mondial d'Observation du Climat (SMOC)}

Le SMOC a été créé dans le cadre de la CCNUCC avec l'objectif de fournir les données nécessaires au suivi du système climatique, la détection du changement climatique et le suivi de la réponse, l'application au développement des économies nationales et la recherche. Le SMOC est un auxiliaire qui encourage, soutient et coordonne les observations mondiales. Il poursuit ses objectifs en se basant sur les activités nationales, en partenariat avec les programmes de l'OMM et autres systèmes mondiaux d'observation (SMOO, SMOT).

Le réseau du SMOC comprend un réseau d'observation en surface, GSN et un réseau d'observation en altitude, GUAN.

\section{Réseau de l'Institut Congolais pour la Conservation de la Nature}

L'Institut Congolais pour la Conservation de la Nature, ICCN en sigle, a reçu la mission d'assurer la protection de la faune et de la flore dans les aires protégées. Parallèlement, elle a la charge de favoriser en ces milieux la recherche et le tourisme dans le respect des principes fondamentaux de la conservation de la nature.

Depuis 2008, l'ICCN a installé des stations climatologiques dans ses airs protégées. Les stations sont dotées des instruments et matériels ci-après : abri météorologique, météorolographe (appareil avec enregistreur), thermomètre à maximum, thermomètre à minimum, pluviomètre et anémomètre. 
Tableau : Réseaux des stations d'observation de l'ICCN dans la province du Haut-Katanga

\begin{tabular}{|l|l|l|l|l|l|}
\hline $\mathbf{N}^{\circ}$ & Nom de la station & $\begin{array}{l}\text { Longiti- } \\
\text { tude }\end{array}$ & latitude & altitude & référence \\
\hline $\mathbf{2 .}$ & KATWE & & & & \\
\hline $\mathbf{3 .}$ & LUSINGA & $27^{\circ} 51 \mathrm{E}$ & $10^{\circ} 33^{\prime} \mathrm{S}$ & $1687 \mathrm{~m}$ & $\begin{array}{l}\text { Parc national de Kundelungu } \\
\text { Kasenga/Katanga }\end{array}$ \\
\hline $\mathbf{4 .}$ & KAYO & $27^{\circ} 12 \mathrm{E}$ & $08^{\circ} 55^{\prime} \mathrm{S}$ & $1789 \mathrm{~m}$ & $\begin{array}{l}\text { Parc national de l'Upemba } \\
\text { Mitwaba/Katanga }\end{array}$ \\
\hline & & $26^{\circ} 02 \mathrm{E}$ & $09^{\circ} 52^{\prime} \mathrm{S}$ & $1330 \mathrm{~m}$ & $\begin{array}{l}\text { Parc national de l'Upemba } \\
\text { Mitwaba/Katanga }\end{array}$ \\
\hline
\end{tabular}

Source : Rapport de mission d'installation de la METTELSAT

\section{Recherche en météorologie et sciences connexes}

Au niveau provincial, l'Université de Lubumbashi, les Instituts d'Enseignement Supérieur et autres centres de recherche devraient jouer un rôle important, dans la recherche sur le climat et la lutte contre le coronavirus. Ce rôle devrait s'étendre à des travaux de fin de Cycle et des mémoires de licence, de DES/DEA et des thèses de doctorat. L'expérience de recherche des modèles, et surtout la réduction et l'adaptation des modèles globaux et régionaux à la réalité de la province du Haut-Katanga devra exister, l'œuvre d'un individu à l'occasion de la préparation de sa thèse ou de son mémoire de DEA ou autres travaux personnels et non une initiative de l'université ou institut d'enseignement ou de recherche.

L'accès à l'internet étant aujourd'hui assez facile, les universités peuvent profiter ainsi de cet outil et ainsi que d'autres réseaux de communication aujourd'hui disponibles pour avoir accès aux produits des divers satellites et même parfois recevoir directement les données ou par le canal de certains Universités partenaires à travers le monde. C'est le cas par exemple de l'ERAIFT qui collabore avec l'Université de MARYLAND et la NASA.

Dans le cadre de la recherche météorologique et climatique, il faudra équiper et renforcer les capacités de recherche de la METTELSAT pour arriver à l'instar d'autres pays Africains tel que le Maroc, à élaborer aussi des modèles de prévision météorologique, climatologique, hydrologique et pour le grand public, adapter à notre pays et à chaque ville sur base des mailles d'observation plus fine.

\section{EDUCATION, FORMATION ET SENSIBILISATION DU PUBLIC}

Conformément aux dispositions des articles 4 et 6 de la Convention-Cadre des Nations Unies sur les Changements Climatiques, les parties sont encouragées à soutenir les actions et initiatives de coopération en matière de l'éducation, de formation et de sensibilisation du 
public dans le domaine des changements climatiques ainsi que la participation la plus large à ce processus, notamment celle des organisations non gouvernementales.

L'objectif général visé consiste à capitaliser les savoirs, les savoirs faire, les connaissances, les expériences et les bonnes pratiques et de développer des actions pertinentes et efficaces de sensibilisation, de communication et de plaidoyer ciblées aux différents groupes d'acteurs et de décideurs pertinents à travers des messages clés et efficaces sur les stratégies à adopter dans le cadre de la lutte contre les changements climatiques et la covid-19 dans la province du Haut-Katanga. Il s'agit plus spécifiquement de :

- Evaluer le niveau général de sensibilisation et de compréhension des enjeux des changements climatiques et de la covid-19 et la politique de l'éducation sur les changements climatiques et la lutte contre la covid-19, la formation et la sensibilisation du public;

- Evaluer le programme national et les efforts déployés par le gouvernement (par exemple, Ministère de l'Education) à l'enseignement primaire, secondaire et supérieur;

- Evaluer les informations disponibles sur la mise en œuvre des campagnes, des programmes et des initiatives d'information publique prévues;

- Evaluer les informations disponibles sur les programmes de formation mis en œuvre ou prévues.

- Evaluer les informations disponibles sur les centres d'information sur les changements climatiques et la lutte contre la covid-19;

- Evaluer les informations disponibles sur le cadre institutionnel et juridique pour la participation du public et l'accès à l'information;

- Evaluer les informations disponibles sur la coopération internationale pour promouvoir l'éducation, la formation et la sensibilisation du public.

\section{Niveau général de sensibilisation}

Plusieurs acteurs et structures sont impliqués dans les actions d'information, de sensibilisation et de communication basée sur le changement climatique et la lutte contre la covid-19.

\section{Secteur étatique}

On notera l'engagement et la détermination de certains services techniques et projets du MEDD qui s'emploient à développer tant soit peu, les actions d'information, sensibilisation et communication du public sur les programmes et actions visant la lutte contre les changements climatiques et la lutte contre la covid-19.

De différents acteurs intervenants dans les programmes et projets du secteur environnement consacrés sur les changements climatiques s'impliquent également au processus.

Le MEDD collabore étroitement avec les organisations de la société civile à travers la plateforme GTCR pour les consultations participatives dans le processus REDD+ et autres initiatives visant les actions basées sur les changements climatiques. 
Dans le cadre de suivi de la mise en œuvre des actions visant l'application de la CCNUCC dans la province du Haut-Katanga, le gouvernement provincial à travers le Ministère de l'Environnement, Conservation de la Nature et Tourisme, devra réaliser sous la conduite de la division de développement durable, les communications provinciales en vue d'évaluer l'état d'avancement des programmes, projets et plans d'actions mis en place dans le contexte de la lutte contre les changements climatiques et la lutte contre la covid-19.

\section{Secteur non étatique}

Les acteurs non étatiques participent et collaborent dans le processus de consultation pour l'élaboration des documents et certains outils des programmes et projets dans le cadre du processus de lutte contre les changements climatiques et la covid-19 dans la province du Haut-Katanga.

Certaines agences des Medias s'engagent pour la création des espaces en vue de l'animation et la diffusion des émissions et messages clés dans les chaines des radios et télévisions aux niveaux provincial et local; l'existence et la présence du top réseau médias des Communicateurs REDD+ demeurent un atout majeur pour impulser une action médiatique efficace et durable.

S'agissant des organisations de la société civile, des structures de base telles que le Comité Provincial de Participation (CPP) et le Comité Local de Développement et de Conservation (CLDC) devraient être créées et constituer une opportunité pour rendre l'information, la sensibilisation et la communication systématiques et fluides auprès du public, surtout au niveau des communautés de base.

Certaines ONG, dans le cadre des projets REDD+, doivent organiser des campagnes de sensibilisation sur les projets et programmes environnementaux, les changements climatiques et la lutte contre la covid-19; ce qui concoure à maximiser positivement les efforts de sensibilisation, d'information et communication du public sur la lutte contre la covid-19.

Enfin, pour la sensibilisation et la mobilisation communautaire, les ONG provinciales et certains projets et programmes devraient utiliser des chaines de radios de proximité à Lubumbashi et à l'intérieur de la province du Haut-Katanga.

\section{PANA}

Le PANA, Programme National d'Adaptation au Changement Climatique en République Démocratique du Congo, par son antenne dans la province du Haut-Katanga, est appelé à intervenir dans le domaine de l'adaptation aux effets et impacts du changement climatique face à la covid-19. Ce processus devra permettre aux populations les plus vulnérables et aux communautés de base les plus exposées de faire face aux conséquences des changements du climat et de lutte contre la covid-19 dans la province du Haut-Katanga.

Ce projet devra développer certaines actions pour la communication et la sensibilisation au bénéfice des populations paysannes dans ses sites d'intervention et pour l'ensemble 
du public. Ce sont des formations, des conférences (tables rondes), des publications, des émissions radiophoniques et télévisées, des documentaires, des foires, etc.

Ces actions d'information et communication au public devraient porter sur les messages suivants:

- Gestion des eaux en milieu rural et fertilité du sol;

- Pratiques agro écologiques;

- Agro météorologie (les changements climatiques, l'adaptation et les alertes précoces dans le secteur agricole);

- Méthodologie pour l'intégration de la dimension des changements climatiques dans les plans de développement provinciaux;

- Changement climatique, adaptation et gestion des risques climatiques;

- Méthodologie pour l'intégration de la dimension de lutte contre la covid-19 dans les plans de développement provinciaux;

- Adaptation de tous les secteurs de la vie à la lutte contre la covid-19;

- Publication des bulletins d'informations sur la lutte contre la covid-19 quotidiens. Il faudra créer un site web et la publication d'un bulletin d'information en ligne.

\section{E. REGARD SUR L'EVOLUTION DE LA PANDEMIE DE CORONAVIRUS DANS LE HAUT-KATANGA}

Depuis le début de l'épidémie déclarée le 10 mars 2020, le cumul des cas est de 14.461, dont 14.460 cas confirmés et 1 cas probable. Au total, il y a eu 352 décès (351 cas confirmés et 1 cas probable) et 12.465 personnes guéries. Le test Covid-19 est gratuit pour tout le monde en République Démocratique du Congo. Cependant, le test des voyageurs est payant à 30 dollars américains. Parmi les provinces touchées, la province du Haut-Katanga compte 401 cas à la date du 12 décembre 2020. Sur les vingt-deux provinces touchées au pays, elle occupe la quatrième place après la ville- province de Kinshasa (11.220 cas); la province du Nord-Kivu (1.190 cas); le Kongo Central (660 cas). A titre de rappel, c'est depuis le 21 mars 2020 que la province du Haut-Katanga avait enregistré le premier cas de la covid-19.

La République Démocratique du Congo a connu trois mois de confinement (allant du mois de mars au mois de juin 2020). Pendant cette période, dans la province du Haut-Katanga, les entreprises minières ont confiné aussi leurs travailleurs. La province du Haut-Katanga compte cent quatre-vingt-cinq titulaires des droits miniers et des carrières ${ }^{16}$. Ainsi, en dépit du fait qu'il y a eu ralentissement dans la commercialisation des produits miniers, la production est restée la même, voire a augmenté malgré la diminution du nombre d'employés ayant travaillé dans des conditions peu acceptables (manque de repos, non-respect

16 République Démocratique du Congo, Ministère des Mines, Cellule Technique de Coordination et de Planification Minière "CTCPM », Répertoire des opérateurs du secteur des Mines et Carrières, Edition 2017, septembre 2018, pp12-25. 
des heures de service, prime de confinement inexistante dans certaines entreprises; là où elle existe, elle est modique, alimentation non appropriée). " Le « coffre-fort souterrain » se vide tandis que les Congolais répètent avec raison et de plus en plus fort qu'ils ne mangent pas les statistiques, ni les belles courbes de production à l'allure ascendante. Ils tiennent avoir des retombées palpables et conséquentes $\gg .{ }^{17}$

\section{La gestion du risque sanitaire}

Un système de gestion du risque fondé sur la surveillance des phénomènes sanitaires et la réactivité des partenaires à impliquer en cas de leur survenue, serait un atout pour atténuer et/ou adapter la santé de la population aux changements climatiques. La gestion du risque devrait être conduite selon une approche multidisciplinaire faisant appel aux secteurs impliqués dans la gestion des phénomènes sanitaires, environnementaux et sociaux : cliniciens, épidémiologistes, entomologistes, biologistes, météorologues, climatologues, géographes, sociologues, etc.

\section{La surveillance épidémiologique}

La province du Haut-Katanga devrait être capable de détecter et d'alerter sur tous les phénomènes sanitaires, y compris les plus inattendus à l'instar de la covid-19. Ceci devrait inciter le gouvernement provincial à promouvoir la surveillance des signes, des syndromes, beaucoup plus sensible, au détriment d'une démarche classique qui vise la surveillance des maladies, mais répondant de moins en moins bien au défi de l'imprévisibilité. La surveillance épidémiologique devrait nécessiter la mise au point des indicateurs spécifiques (humains, vétérinaires, environnementaux,...) et des seuils d'alerte qui devraient permettre de mieux appréhender les impacts des changements climatiques sur la santé de la population, en prenant en compte l'extrême rapidité de diffusion de la covid-19, maladie transmissible, essentiellement du fait des voyages aériens et terrestres, et l'extrême sensibilité des opinions publiques, et donc des dirigeants.

\section{Formulation d'un programme d'actions prioritaires}

Un programme d'actions prioritaires en matière de santé et d'action sociale, spécifique aux changements climatiques, devrait être mis en place dans l'optique de l'atteinte des objectifs généraux tels que l'amélioration de l'accès de la population aux services de santé publics et communautaires pour les maladies infectieuses et les maladies chroniques non transmissibles (diabète sucré, hypertension artérielle, maladies cardiovasculaires, maladies rénales) dont la fréquence d'occurrence est liée aux changements climatiques. En outre, il faudrait l'organisation d'une prévision médico-météorologique assortie d'un système

17 R., NGOY MUSHILA, Les défis de la Gécamines, sanctuaire du népotisme ou géant minier performant?, S.L., Première Edition, 2014, p.162. 
d'alerte précoce adapté aux changements climatiques dans la province du Haut-Katanga et la création d'un centre multidisciplinaire de recherches sur le climat et la santé.

\section{Mesures diverses d'adaptations}

- Renforcement des capacités professionnelles du personnel de santé;

- Identification et destruction des repaires des agents pathogènes;

- Organisation des actions préventives contre les maladies vectorielles;

- Renforcement du système d'assainissement;

- Organisation de l'éducation, de la formation et de la sensibilisation de la population;

- Amélioration du système d'alimentation;

- La popularisation de l'utilisation des grilles anti-moustiques;

- L'utilisation de moustiquaires imprégnées;

- L'éradication des gîtes de moustiques;

- Renforcement des capacités nutritionnelles de la population;

- La lutte contre l'exclusion sociale et la promotion communautaire;

- Cartographie des zones d'habitat à risque climatiques;

- Institutionnaliser la coordination de gestion des opérations face aux changements climatiques.

\section{Dans le secteur minier}

L'extraction minière est une activité à forte intensité écologique dont les externalités négatives ne sont plus à démontrer. Le développement des projets miniers constitue à ce titre un facteur de risque environnemental non négligeable pour les communautés locales, en particulier dans les localités minières. Au cours des dernières décennies, il est fait état de nombreux impacts environnementaux de l'activité minière dans les provinces à forte dépendance minière, notamment le Haut-Katanga, dont la prise en charge effective par les entreprises minières où l'Etat pose de nombreux problèmes, liés à la délimitation des responsabilités entre les parties.

Concernant les études d'impacts environnemental et social, il est à mentionner que :

- Tous les Permis et Autorisations d'exploitation ou de recherche sont assujettis à une quelconque obligation environnementale de la part du demandeur. Les études d'impacts environnementaux (EIE) sont requises en vue de l'obtention de tous les permis d'exploitation et doivent être accompagnées du Plan de Gestion Environnemental de Projet (PGEP) qui décrit la mise en œuvre des mesures d'atténuation, de prévention et de réhabilitation du site après fermeture. Dans le cas des permis de recherche, le dépôt d'un Plan d'Atténuation et de Réhabilitation (PAR) est exigé, après l'octroi du permis, comme condition au commencement des travaux;

- Les opérations minières existantes, et dont le permis d'exploitation doit être transformé au moment de l'entrée en vigueur du Règlement Minier, sont tenues de soumettre 
un Plan d'Ajustement Environnemental (PAE) dans les douze mois suivant l'entrée en vigueur du Règlement Minier lorsque la durée non échue dudit permis est de deux ans ou plus;

- Les opérations minières artisanales, qui constituent toujours un sérieux problème environnemental où qu'elles se trouvent, sont également visées et doivent soumettre avec leur demande de permis d'exploitation un Engagement de Protection Environnementale qui s'inscrit à l'intérieur du Code de Conduite de l'Exploitant Artisanal.

Cependant, malgré l'existence de l'arsenal réglementaire et institutionnel, la gestion des impacts environnementaux inhérents à l'activité minière reste encore très limitée. En réalité, à l'exception des impacts directs générés par les entreprises minières en activité dans leur zone d'implantation, il est quasiment impossible d'effectuer une évaluation précise des dommages environnementaux générés par l'industrie minière dans la province du Haut-Katanga.

A ce jour, aucun seuil de rejet admis dans l'environnement (émissions atmosphériques, effluents liquides etc.) n'a jusque-là été défini et les seules exigences auxquelles doivent se soumettre les entreprises minières se limitent à la réalisation d'Etudes d'Impact Environnemental et Social (EIES). Les EIES ont pour objectif d'identifier, d'analyser et d'évaluer les impacts et les mesures de réduction, d'atténuation et de compensation, et déterminent comme résultats des travaux d'investissement spécifiques, des Plans de Gestion Environnementale et Sociale (PGES), des Plans de Réhabilitation et de Fermeture (PRF) et des Plans de Relocalisation. Elles constituent désormais une étape préliminaire des phases de recherche et de prospection, et un moyen d'anticiper les impacts notoires générés par les différentes phases de l'exploitation minière. Les EIES sont commanditées et suivies par les entreprises minières elles-mêmes et leur évaluation est souvent rendue difficile en raison du faible niveau de qualification du personnel local.

Les déplacements des populations riveraines ont été réalisés pour la plupart, sans une étude socio-économique préalable qui devrait permettre d'évaluer le patrimoine des populations affectées et de déterminer de façon précise le niveau de compensation et d'indemnisation des biens perdus. Par ailleurs les populations déplacées ont très souvent été réinstallées sur des sites dont la capacité d'accueil est largement insuffisante par rapport aux besoins.

Sur le plan technologique, les impacts environnementaux et sociaux spécifiques du secteur sont évalués dans le cadre des études d'avant-projets détaillés. Ces évaluations tiennent compte et précisent les actions environnementales et sociales à entreprendre, notamment celles relatives à l'ouverture, à l'exploitation des sites miniers, à la réhabilitation des habitats naturels dégradés, etc. En plus, il est proposé de réaliser des études thématiques (contrôle qualitatif de l'état de contamination des ressources en eau, inventaires, cartographie, etc.) en vue de disposer d'une situation de référence, de banque de données environnementales et sociales pour le secteur, de guides sectoriels et procédures techniques et de bonnes pratiques environnementales et sociales.

Tous les intervenants dans le secteur minier (le Président de la République, le Ministre des mines, le Gouverneur de province, le Chef de division provinciale des mines, le 
Cadastre minier, la Direction de géologie, la Direction des mines, le Service de protection de l'environnement minier) ainsi que toutes les parties prenantes au développement durable devraient exécuter loyalement leurs tâches.

La protection de l'environnement occupe indéniablement une place de choix dans la législation minière de la RDC. C'est dans ce cadre que le code minier et son règlement subordonnent l'octroi des droits miniers à l'obtention préalable de l'approbation des études d'impacts environnementaux (EIE), d'un plan de gestion environnemental du projet minier (PGEP), ainsi qu'un plan d'atténuation et de réhabilitation (PAR) auprès des services techniques compétents. La pertinence de ces études vise, d'une part, la mise en œuvre des mesures de prévention relatives à la protection de l'environnement et, d'autre part, à la mise en place de mesures d'atténuation et de réhabilitation des risques de dégradation de l'environnement après la fermeture du site minier.

Il est discutable d'aborder l'analyse des défis liés à la dégradation de l'environnement par les entreprises chinoises au Katanga dans un contexte où aucune d'elles n'a mené les études requises par la législation de la RDC, ni, encore moins publié les résultats desdites études. La réalisation préalable de ces études aurait exigé que ces compagnies prennent des mesures de manière à limiter les impacts négatifs majeurs de leurs activités sur l'environnement et sur les communautés locales. Bien que la législation minière en vigueur en RDC fasse de ces études un préalable, sinon une obligation, pour la jouissance des droits ou titres miniers, notamment, tels que précisés dans le code minier: le Permis de Recherches, le Permis d'Exploitation, le Permis d'Exploitation de petite mine et le permis de recherches des rejets, lesquels sont constatés par le Certificat de Recherches, le Certificat d'Exploitation, le Certificat d'Exploitation de Petite Mine et le Certificat d'Exploitation des Rejets, ces études ne sont généralement pas effectuées, ni vérifiées.

A Lubumbashi, à Likasi, et leurs environs, la construction des unités industrielles de traitement de minerais et l'installation des comptoirs d'achat et de dépôts de minerais appartenant aux entreprises chinoises installées dans les quartiers résidentiels, engendrent des risques réels pour les habitants desdits quartiers. Les règles scientifiquement indiquées en la matière, ainsi que la réglementation minière relative à l'entreposage, au transport et à la commercialisation des produits miniers sont systématiquement négligées. Certaines principales avenues des villes citées ci-dessus, servent à l'installation des comptoirs d'achat et à l'entreposage des minerais. Ainsi, l'absence d'études environnementales traduit l'absence de l'évaluation des dommages causés sur l'environnement d'une part, et celle des mesures susceptibles non seulement de réparer les dommages probables causés sur l'environnement, mais aussi d'en limiter les effets d'autre part.

\section{CONCLUSION}

La lutte contre le réchauffement climatique à l'ère de la covid-19 doit s'inscrire dans le développement durable de la province du Haut-Katanga. Il serait indispensable, entre autres, de mettre en évidence la disponibilité éventuelle d'une charte, d'un label ou d'une 
certification DD/RSE au sein de chaque entreprise; d'évaluer le degré d'intégration des principes du développement durable aux stratégies d'affaires des entreprises minières; et enfin, de relever les différents facteurs incitant ou non ces entreprises à l'adoption d'une démarche DD/RSE. Il s'agit notamment de la disponibilité d'un label ou d'une certification RSE intégrant les dimensions environnementale et sociale du développement durable.

Il serait mieux d'évaluer le degré d'engagement de toutes les entreprises minières dans la mise en œuvre de politiques ou démarches DD/RSE. A ce titre, il faudrait mettre l'accent, respectivement, sur l'intégration des principes du développement durable au management des ressources humaines à savoir: la vulgarisation du concept au près des employés et les canaux de diffusion utilisés, les pratiques DD/RSE des entreprises en faveur des employés, l'intégration des fondamentaux du développement durable aux décisions en matière de gestion des ressources humaines; la gestion de l'eau, de l'énergie et des émissions de gaz; l'application des principes du développement durable au processus de production; l'intégration des principes du développement durable aux choix et décisions d'investissement, et les perspectives quant à l'adoption d'une démarche $\mathrm{DD} / \mathrm{RSE}$.

En outre, s'ajoutent les impératifs ci-après : la sensibilisation des employés au développement durable (formation en interne, conférences et colloques; ateliers et séminaires...); le respect des normes; depuis quelques années, on assiste à un foisonnement des normes internationales visant la promotion du développement durable dans différents secteurs d'activité, notamment le secteur minier, portant généralement sur les dimensions économique, environnementale et sociale. A ce titre, les entreprises minières sont appelées à adopter et à respecter entre autres: la « Déclaration de Principe Tripartite sur les Entreprises Multinationales et la Politique Sociale $»^{18}$, l'Organisation Internationale du Travail (OIT) ${ }^{19}$, les « Principes Directeurs de l'OCDE » à l'intention des entreprises multinationales ${ }^{20}$, les 10 principes de l'initiative « Global Compact» de l'Organisation des Nations Unies (ONU) ${ }^{21}$.

Ces normes visent pour l'essentiel l'abolition du travail forcé, la promotion de la liberté syndicale et le respect des droits de négociations collectives, le respect des droits de l'homme et des normes du travail, la promotion du développement durable, la protection de l'environnement et la lutte contre la corruption, l'incitation à la publication d'informations sur la politique sociale des firmes, la protection des consommateurs etc.

\section{REFERENCES BIBLIOGRAPHIQUES}

1. Bélanger, 2002.

2. Bourque et Simonet, 2007.

18 https://www.reportingrse.org/oit-p-40.html. consulté le 25 février 2017.

19 https://www.oecd.org/fr/daf/inv/mne/principesdirecteursdelocdealintentiondesentreprisesmultinati onales.htm consulté le 25 février 2017.

20 https://www.unglobalcompact.org, consulté le 25 février 2017.

21 https://www.reportingrse.org/industriesextractives-p-163.html\#pave79, consulté le 25 février 2017. 
3. Bush, E. et G. Flato, rapport sur l'environnement et le changement climatique, Ottawa, Ontario, 2019, p. 7-23.

4. Denis, 2010.

5. Environnement Canada, 2002.

6. Giguère, 2009.

7. IOCHA and IDMC, 2009.

8. R., NGOY MUSHILA, lLes défis de la Gécamines, sanctuaire du népotisme ou géant minier performant?, S.L., Première Edition, 2014.

9. Rapport de mission d'installation de la METTELSAT.

10. République Démocratique du Congo, Ministère de l'Environnement, Conservation de la Nature et Tourisme, seconde communication nationale à la convention cadre sur les changements climatiques, données de l'inventaire des émissions et de séquestration des gaz à effet de serre (GES) de chaque secteur socio-économique entre 1999 et 2004 ainsi qu'une étude de la vulnérabilité du pays par rapport aux changements climatiques et des mesures d'adaptation des secteurs forêts, agriculture, zone côtière, eau et santé, p.59 (212).

11. République Démocratique du Congo, Ministère des Mines, Cellule Technique de Coordination et de Planification Minière "CTCPM », Répertoire des opérateurs $d u$ secteur des Mines et Carrières, Edition 2017, septembre 2018, pp12-25.

12. Roy, 2002.

13. Santé Canada, 2008.

14. https://www.reportingrse.org/oit-p-40.html.

15. https://www.oecd.org/fr/daf/inv/mne/principesdirecteursdelocdealintentiondesentrepri sesmultinationaleshtm.

16. https://www.unglobalcompact.org, consulté le 25 février 2017.

17. https://www.reportingrse.org/ industries extractives-p-163.html\#pave79. 